\title{
E-Branding: A New Approach for Promoting Polyhouse
}

\author{
Sudeepthi Govathoti ${ }^{1}$, Deepthi Kamidi ${ }^{2}$, N.Thirupathi Rao ${ }^{3}$, \\ Debnath Bhattacharyya ${ }^{3}$ and Hye-jin Kim ${ }^{4 *}$ \\ ${ }^{1}$ Anurag Group of Institutions, Hyderabad, India \\ ${ }^{2}$ K.L. University, Vijayawada, India \\ ${ }^{3}$ Dept. of Computer Science \& Engineering, Vignan's Institute of Information \\ Technology, Visakhapatnam-530049, India \\ ${ }^{4}$ Business Administration Research Institute, \\ Sungshin W. University, 2, Bomun-ro 34da-gil, Seongbuk-gu, Seoul \\ ${ }^{1}$ sudeepthicse@cvsr.ac.in, ${ }^{2}$ deepukamidi@gmail.com, ${ }^{3}$ nakkathiru@gmail.com, \\ 32debnathb@gmail.com, 4*hyejinaa@daum.net
}

\begin{abstract}
Polyhouse is a structure which is constructed to provide secured and controlled environment for the proper growth of the plant. The polyhouse cultivation method provides high growth and improved productivity. To achieve proper crop management it is necessary to collect the correct information to make decisions. In the current work we considered and proposed a framework which could able to collect the information related to polyhouse atmosphere and harvest status and manage the polyhouse automatically based on the collected information. The polyhouse information is collected from different resources by using sampling method. Sampling method contains different strategies that are Simple random, stratified random, Stratified-systematic, Judgmental. Each method identifies the data in different location and collects the data. The collected data to be stored in the mongo database. The data base utilize data cleansing, data scarping, data integration and data wrangling process to arrange the data for relevant user. It helps to fix the data in specific manner in the database. Finally, the user can retrieve the data using the Latent Indexing Algorithm to retrieve the data in specific manner. It helps the system to forecast and take steps on situations intended for completely guarded climatic circumstances. So that farmers can access the relevant information using IoT. After the cultivation process the farmers can also marketing their product directly through IoT.
\end{abstract}

Keywords: Polyhouse, Internet of Things, Latent Indexing Algorithm, Data wrangling

\section{Introduction}

World Wide net pool is Associate in nursing extension of linguistics net. The linguistics net describes 2 things. That's combination and sequence of knowledge from varied sources, and it's chiefly targeting the swap of documents. The linguistics net additionally concerns language for recording however the info relates to world objects. That enables someone, or a machine, to induce in one table, and so shift to a different table that are connected by the wired network [1]. Generally, the linguistics net isn't a machine-process in a position. It cannot be traversed by net agents or applications. In linguistics net the knowledge are taken from completely different sources cannot be without delay accessed and even supposing everybody will say something concerning something, only a few folks are literally detected. We tend to solve the matter of linguistics net by doing a little of its basic assumption [2].

Received (May 7, 2017), Review Result (August 23, 2017), Accepted (September 11, 2017)

${ }^{*}$ Corresponding Author 
IoT comes below the extending current web and providing association between devices is usually means the IoT[3]. IOT based mostly good environments have some distinctive characteristics like, it's some prediction capabilities, freshly increased services, remote watching and higher cognitive process capabilities. The conception of IoT is applied to any or all aspects of transportation, like assortment of knowledge associated with rider investigating, geo services, good ticketing, and communication. IT result will assist in connecting each conveyance part, and furnishes the technical tools to attach sensors and different devices to that infrastructure [4].

The device layer is used for sensing environment collecting data and controlling home appliances. Network layer connects the device layer to the application layer. The data storage and retrieval are done by using this Cloud management layer. Application layer providing services to the end user and these services also provided to the third parties [5]. Also IOT has some features like security, provisioning, interoperability, latency, massive number of devices, scalability. IOT need standardized protocols to enable all devices to communicate with one another with various feature. The protocols are, Restful HTTP :( Representational state transfer) it is work on top of HTTP and uses the rest principles at a time. Restful HTTP is a lightweight and simple HTTP format easy to implement. COAP is generalized web transfer protocol for use with constraint devices and networks. Message queue telemetry transport is a publish/subscribe extremely simple light weight messaging protocol. The adaptable messaging and presence protocol is an application profile of the extensible mark-up language. XMPP extension through multiple XMPP extension protocols. The advantages of IOT are, reduce transport cost, predictability of prices on the past data analytics and the current market condition and also reduce number of middle hops and agents.

Agriculture is the backbone of India's economic activity. Because, cultivation is crucial thing to people and it for a food security. Cultivation also helps to human beings to increase the main food grains and also increase the right animals (the rights of animals to live free from human exploitation and abuse) with conformity to surrounding aspects. India has become an economical possible in the world. Our cultivation productivity must equal to other countries which are still rated as economic power of the world. Nowadays, Polyhouse is popularly used in many countries especially temperature ones such as Japan, Mexico, the northern lands, Korea, France growing vegetables in polyhouse research was conducted at May 2007.The main aim of this research was building a suitable polyhouse in hot climate regions. The polyhouse are also giving a good environment so the plants are growing properly and also produced good healthy foods [6]. There are many advantages because the plants developed in a secured surrounding like a polyhouse. Also it produces the yield diseases and the required environments are used to increased productivity and can be met by adopting polyhouse cultivation method [7]. The Polyhouse method is a new technique in cultivation achieved comfort in agricultural India and can be strongly employed for slot areas of agriculture.

Polyhouse farming and still because the different ways of secured atmosphere agriculture has been derived to create a services small climate, that favors the crop production, can be attainable throughout the year or a part of the year pro re nata. The encompassing parameter ordinarily controlled the temperature, commonly giving heat to manage the cold conditions [8]. The condition is most vital factor in polyhouse. But environments condition additionally includes the cooling to scale back excessive temperatures, management light-weight either shading or adding further light, dioxide levels, relative wet, water, plant nutrients and bug management. Processed system are fashionable polyhouse additionally its normal technology with it's a continuing enhancements because the technology advances. Environmental parameters like weather are required to take care of these processed management systems, wherever the system is operated manually and/or mechanically. Atmosphere management technology remains restricted, chiefly as a result of its expensive. Therefore, the development of atmosphere 
watching and system for comprehensive polyhouse production is important. The electrical device and information acquisition (DAQ) card are wont to watching and dominant the temperature, wet and lightweight signal that are coming back from polyhouse. The electrical device Associate in nursing information acquisition card is act like an interface to the pc.

The methods are data acquisition, temperature sensor, humidity sensor, light intensity control, solid state relay. In data acquisition the Polyhouse monitoring system developed, the DAQ card used is NI PCI 6251 from National Instruments. The main features of this DAQ card includes a sampling rate $1.25 \mathrm{MS} / \mathrm{s}, 16$ analog inputs having 16 bit resolution, 2 analog outputs, 24 digital input outputs channels, Maximum the input ranges are from $10 \mathrm{~V}$, On board memory 4095 samples, Input impedance $>10$ activity in coordinate with $100 \mathrm{pF}$ etc. One of the types of resistance thermometer is the type of resistance thermometer also known as the resistance temperature detector. Soil and air temperature are monitored by using this sensor. In resistive humidity sensor the changes occur in electrical resistance of a material because of humidity. The humidity sensor with water circulating pump are used to control the humidity of air. So here we used the relative moisture of air. The resistive humidity sensor is one of the type of watering system and it is warn to find out the humidity of air and it is less then motor pump and then water is giving to the plant.

The enormous heats that are prepared by the shinning tubes and that are unacceptable in most instance Fluorescent tubes are useful as the exclusive source of light. LDR is used by us for monitoring of light intensity in polyhouse. Stable case communicate control the physical devices like pump, fan and heater, a thermistor based Stable case communicate is used. One of the digital outputs of the stable case communicate are triggered by the DAQ card and thus control the energizing coil of the contactor. The solid state relay, used, has $330 \mathrm{~V}$ ac, $10 \mathrm{~A}$ rating on the output side and it has a triggering voltage rating of 3 to $32 \mathrm{~V}$ dc [9]. Nowadays the recorded lodging and restaurants are using polyhouse to receive best kind of vegetables and fruits from both periodic and aperiodic season to maintain their influence. We can expect large scale of profit from polyhouse farming [10].

\section{Related Work}

M. Pawar et.al., [21] focuses on observance the environment parameters of hi-tech polyhouse the Wireless detectors Network (WSN). The Wireless Sensor Node are that the heart of universal field. Moreover, embedded technology placed the sector of microcontroller is original and most reliable. Therefore, utilizing established innovation, in light-weight of AVR AT mega 8L microcontroller, the Wireless detector Node created and dead for improvement of WSN for polyhouse applications. In like manner, in sight of AN inserted innovation and also the RF module Zigbee an overseas adult male hub is made public concerning extremely encouraging AVR ATmega8L microcontroller and dead for WSN improvement. To upgrade trim yield one has to offer controlled atmosphere to the harvest.

Basant Maheshwari et. al., [27], The Australian greenhouse industry is essentially ruled by minimal effort hydroponic greenhouses for conveyance of water and supplements to plants to grow an assortment of vegetable yields including cucumber and tomato. The water which is the supplement rich drainage water from these greenhouses is for the most part discharged into the nearby environment creating contamination concerns. The study demonstrated that the recycling of the drainage water brought about a 33\% decrease in potable water utilized for water system as a part of cucumber generation. The drainage water contained $59 \%$ connected $\mathrm{N}, 25 \%$ connected $\mathrm{P}$ and $55 \%$ connected $\mathrm{K}$ and represented the potential for nutrient recuperation and generation cost investment funds through the reuse of drainage water. This contextual analysis shows that some moderately straightforward changes in water system hones inside greenhouse frameworks to reuse 
drainage water can significantly enhance supportability of ease hydroponic greenhouses and minimize the natural impression of the greenhouse industry.

P. Kalia et al., [28], the off-season nursery creation was discovered productive under low cost polyhouse of 50 meter square zone. Essentially, development of high value offseason vegetables under low cost ensured structures was found a reasonable innovation for developing vegetables effectively amid winter with impermanent insurance from November to February. The create from ensured structures was off-season and consequently fetched higher costs in the market. Under low amount secured structure of $50 \mathrm{~m} 2$ a total of Rs. 9500/ - was get in first year and around Rs. 24,000/ - from second year onwards by raising offseason nursery. Similarly, a net profit of Rs.15, 000/-and $59,500 /$ - can be earned by growing French bean and gherkins in first year and Rs. 43,500/- and Rs.59, 000/-second year onward from $1000 \mathrm{~m} 2$ area respectively. Therefore, to enhance income and to ensure nutritional security of the small and marginal farmers, off -season nursery as well as vegetables cultivation under minimal effort poly houses is create to be economical and profitable enterprise.

S. J. Singh et.al., [29] Surveys of polyhouse grown Capsicum in Pune for virus diseases was carried during 2000-2001. A variety of symptoms were observed in these polyhouse grown Capsicum plants. The Common symptoms observed were: stunting, generally yellowing of plants, leaf deformation, reduced size of newly developed leaves, systemic mosaic mottling, vein-banding, vein clearing, blistering and file form leaves. Symptoms on fruit were reduction in size, discoloration, strand blistering. Occurrence off our viruses viz. cucumber mosaiccucumo virus(CMV), Potato(PVY), tobacco etch(TEV) and pepper venial mottle(PVMV) pot viruses were recorded on the basis of symptom diagnosis, mechanical transmission, reaction on selected test plants and serological relationship by DAS-EUSA, the most prevalent virus was CMV followed by PVY, PVMV and TEV. The study also revealed that the viruses could infect Capsicum plants alone or in combination. PVMV and TEV were detected for the first time in Capsicum from Maharashtra.

\section{Proposed Methodology}

In this paper we propose the Poly houses information that are stored in the database and also retrieved the information by relevant users. As shown in Figure 3.7, the proposed technique consists of the following blocks. The Poly house information are collected by particular method to be followed as well as the data are being collected. Then the collected information is stored in the multi-dimensional database. The multi-dimensional database using different scheme to stored and provide the user required information. Finally, the information will be retrieved by user using efficient algorithm.

The main modules are data collection strategies of poly house information, data collection, Data stored in the mongo database, finally the information retrieval. Below Figure 1 show that the work flow of the proposed method.

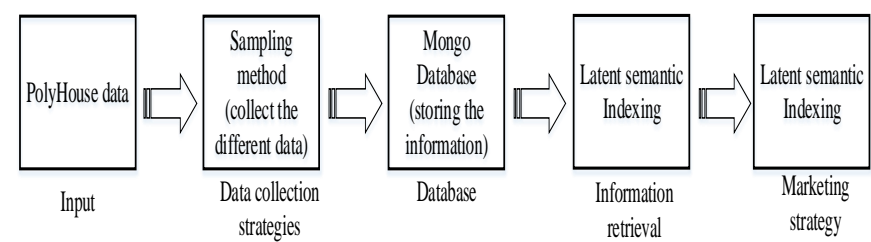

Figure 1. Block Diagram for Proposed System

\subsection{Data Collection}

In this proposed method poly house information is collected from various resources. In previous block describe the how the data to be collected and that include the properties of 
the data. The various levels and fields of data to be collected by using sampling strategies. Then the collected information is arranged in the data collection block.

In example the data collection for any vegetable that relevant area to be located and vegetable growing temperature, property of the vegetable to be collected. The properties of the data represents the vegetable growing temperature, atmosphere, humidity of the vegetable and the required pesticides of the vegetable, amount of soil using in the plant that to be included. In this each properties of the data to be important for the collecting the data because of the user required any kind of information so the data collected in every field with any properties of the plant.

Different strategies available for collecting the data are as follows,

\subsection{Sampling Method}

Data collection is the way toward social event and measuring data, which then provide the power to do something for relevant result. Even though the experiment design are characterized by the limitations of the agriculture and their properties. Still, traditional experimental designs are characterized by limitations and accordingly may not represent a real cropping system. In this field experiment effects and data collections are depend upon the sampling method. The sampling method described the how to collect the data and the different locations of the data and their properties to be viewed. The sample method provides the properties of the poly house method such as temperature, location hectares, and weather condition that to be included. The sampling method contains some strategies for collecting the data that are, Simple random, stratified random, stratified systematic and judgemental. General example for sampling strategies is shown in Figure 3.2 to 3.6.

1. Simple random: Locations are randomly selected that means the types of plant to be located and their properties also located.

2. Stratified random: The chose area is separated into a few territories as indicated by its attributes and examining areas are chosen haphazardly and after that mixed, decreasing the impact of nearby assorted variety.

3. Stratified-systematic: The isolated territory of every cell is additionally separated into littler cells to attempt to defeat the predisposition presented by orderly examining.

4. Judgmental: At long last they chose testing areas are chosen in view of perception of a particular issue and their properties.

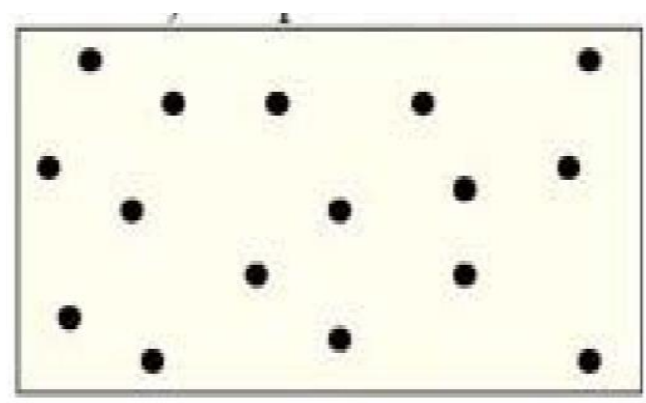

Figure 2. Simple Random 


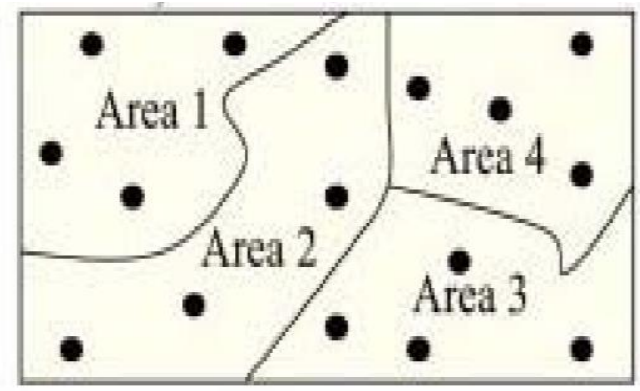

Figure 3 Stratified Random

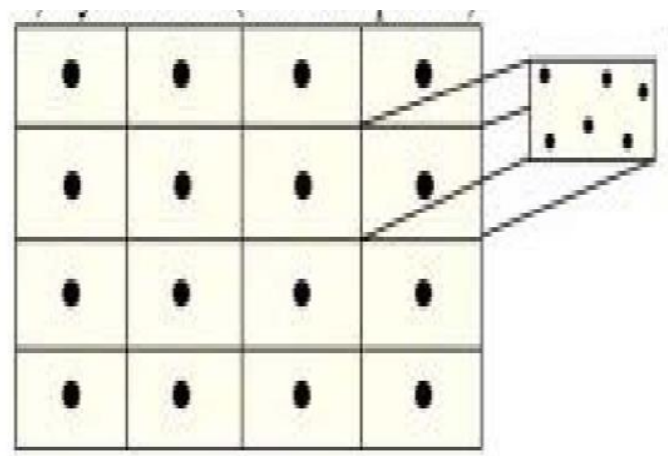

Figure 4. Systematic (cell composite)

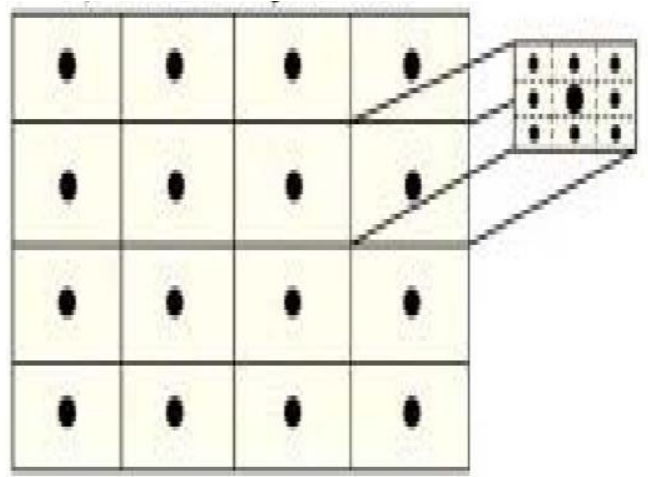

Figure 5. Stratified Systematic

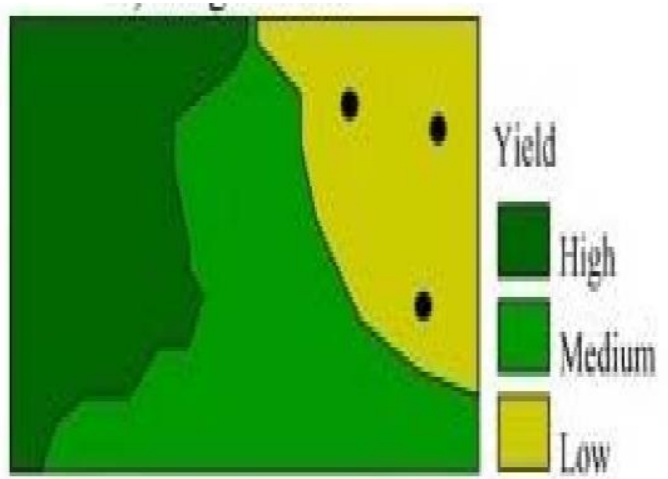

Figure 6. Judgemental 
Sample collection involves the data collection strategies and the way of approach in the field. Each data in the field having specific properties and the measuring experiments. Even though the collection of data not to be arranged that viewed in the randomized manner. So the users need data not to be picked in the normal format. So using different collection strategies for collecting the data in specific manner and then the data to be stored in the data base that are explain briefly in the following subsection.

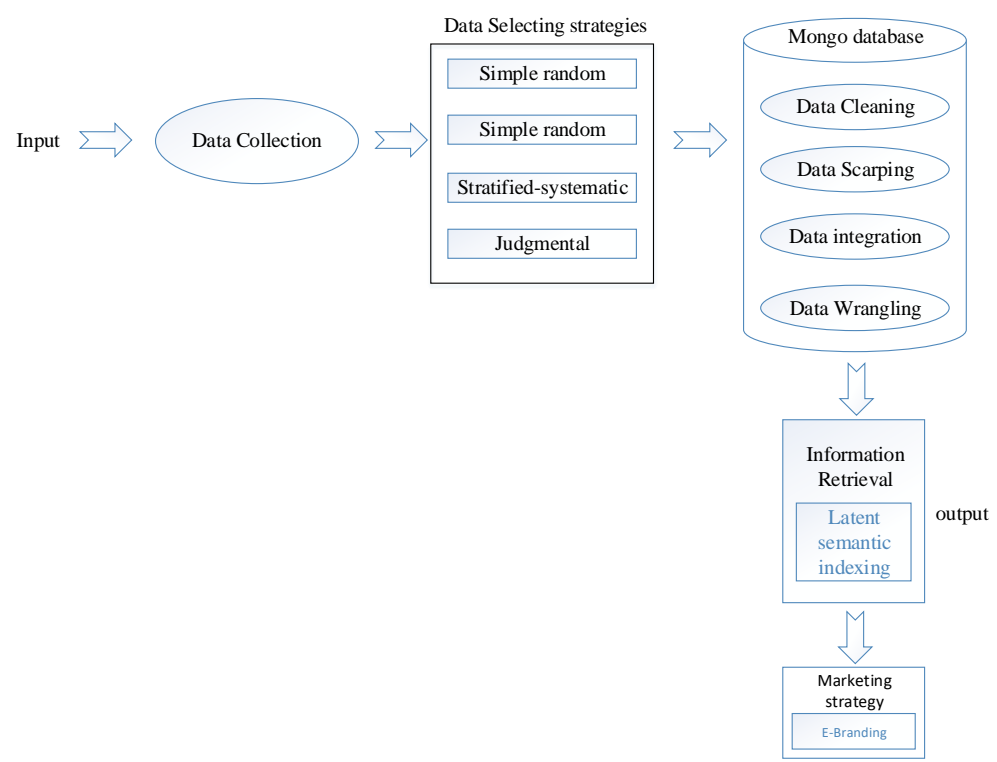

Figure 7. Proposed Methodology

\section{Mongo Database}

Mongo decibel documents influence to possess all information for a given record during a single document, supposing during an electronic database service $\}$ information for a given record is sometimes unfold across several tables. During a computer database, the info model would contain multiple tables like classes, Tags, Users, Comments and Articles shown in fig three. In MongoDB the info can be designed as 2 collections, one for users, and also the different for articles. In every diary document there may be multiple comments, multiple tags, and multiple classes, every expressed as AN embedded array.

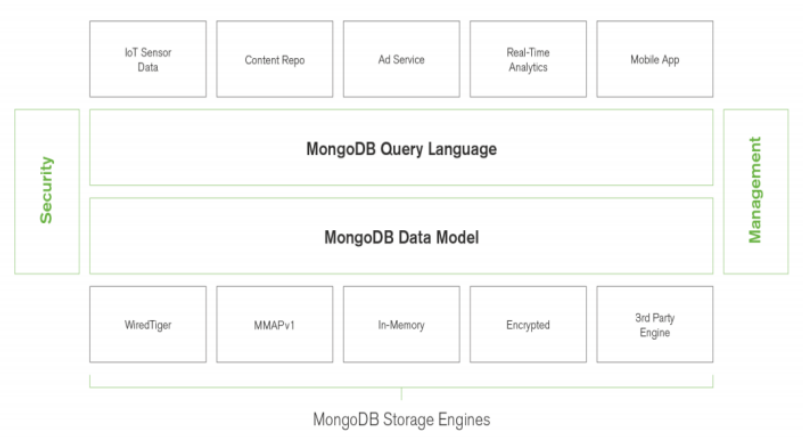

Figure 8. Mongo DB Data Model

\subsection{Data as Documents}

Mongo DB archives impact to have all information for a given record in a solitary report, assuming in a social database data for a given record is generally spread crosswise 
over many tables. In a social database, the information model would contain various tables, for example, Categories, Tags, Users, Comments and Articles appeared in Figure 3.9. In MongoDB the information could be composed as two accumulations, one for clients, and the other for articles. In each blog archive there may be various remarks, numerous labels, and different classifications, each communicated as an inserted cluster.

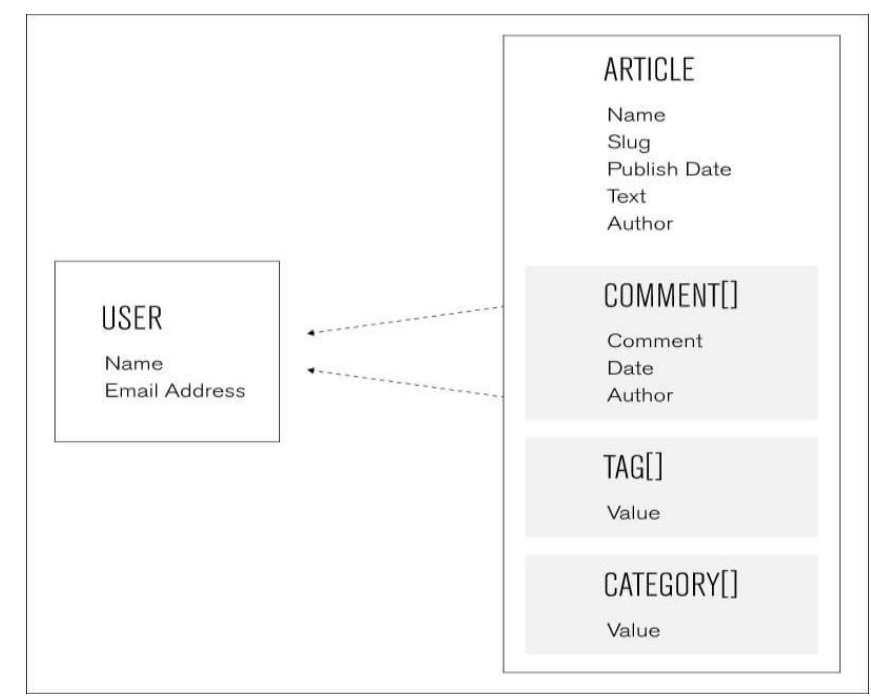

Figure 9. Data as Documents

Data as documents is simple for developers, quicker for users. In our planned system the classified information may be hold on in Mongolian monetary unit info as rows and columns. Every individual info holds on in every field in info. That the info may be retrieved from Mongolian monetary unit info specifically. However, the user would like info are retrieved from the info victimization economical algorithmic rule techniques that in brief delineated as given sub section.

\subsection{Data Cleansing}

Data cleansing is that the method of investigation and removing corrupt or inaccurate records from a record set, table, or info. Conjointly it refers to finding the unfinished, irrelevant, inaccurate elements of the information and so replacement, modifying, or deleting the relevant information. Once playing the cleansing method, every dataset clearing the noise which per different similar dataset. The contradictory information detected or removed by cleansing method which will are elicited by user entry errors, corruption in transmission or storage, totally different information wordbook definitions of comparable entities in several stores. While not utilizing information cleansing strategy within the information base system that happens the some problems that are, Lack of quality of the information, Loss of trust, declined the user base, Loss of business support and funding.

\subsection{Data Scarping}

Data scraping is additionally referred to as net scarping that used for extracting information from websites. In our planned system the information scarping means that the relevant data solely extracted by the method. Unremarkably the information to be corrupted that happens therefore some problems so overcome by these problems victimization data cleansing method. Once playing the cleansing method we have a tendency to apply the information scarping for data extraction. The scarping method keeps the interchange formats and protocols are generally firmly structured, well- 
documented, simply verify, and keep unclearness to a minimum. That the information not is appropriate for human clear one. Information scraping usually involves neglecting binary information (usually pictures or multimedia system data), show format, redundant labels, redundant comment and different info that is either moot or compel automatic process.

\subsection{Data Integration}

Data integration involves combining information that are collected from I totally different sources and providing users with a helpful read of those information. During this our planned system we have a tendency to victimization information integration for desegregation the information from relevant user. the information scarping wont to extract the relevant data from cleansing method at that time the information are integrated by current field of the information. During this method given the every information set having connected data properties and their demand supported the user would like. Information integration seems with increasing density because the size and also the have to be compelled to share existing information collapse. It's become the main target of user would like of the actual product and their properties. Conjointly it integrates the every of the info with relevant information regarding it during a theoretical method. Primarily the idea of knowledge integration forms a set of info theory and formalizes the underlying ideas of the matter in first-order logic. Applying the theories provides indications on the expedience and issue of knowledge integration.

\subsection{Data Wrangling}

Data wrangle is that the method of changing or mapping knowledge from one format into another format that permits for additional convenient consumption of the information with the assistance of semi-automated tools. During this our projected system victimisation this knowledge wrangle method is employed for the various format of the information to be amendment with human clear one. The information scarping everchanging the information with completely different format when integrate the information are going to be the input of the information wrangle method. Therefore, the method changing the information to human clear one. This might embrace any mugging, knowledge visualisation, knowledge aggregation, coaching an applied math model, still as several different potential uses. Currently the accelerated growth of the net victimisation multiple techniques can become progressively necessary within the organization of the growing amounts of information offered. There the information wrangle is that the technique for data changing. In an exceedingly wrangle method, knowledge wrangler is that the person playing the wrangle method. The information wrangler refers to an individual answerable for gathering and organizing dissimilar data sets collected by several field of information set or assembling the information to be changing within the user clear format. The term "Data Wrangler" that's referred because the best correlation to software engineer for code for somebody operating with knowledge.

\subsection{Latent Semantic Indexing}

Latent linguistics categorisation (LSI) is a categorisation and retrieval methodology that victimisation one mathematical technique that known as singular price decomposition (SVD). The singular price decomposition wont to establish the patterns within the relationships between the terms and dataset contained in an indeterminate assortment of text. LSI relies on the principle that words that are utilized in identical contexts favour to own similar meanings. In our projected system we have a tendency to be LSI system that providing needed info for user within the retrieving method. In example user needed the any arrange details means the system providing the plant connected relevant info such tomato is that the plant that having explicit temperature for growth, pesticides, water 
accumulation, humidity, atmospheric condition, etc. These styles of info are going to be provided by victimisation latent linguistics categorisation owing to its ability to associate semantically connected terms that are inherent in an exceedingly assortment of text for user.

\subsection{Benefits of LSI}

LSI overcomes 2 of the foremost problematic restraint of mathematician keyword queries that square measure, the multiple words that have same that means and words or that has over one meaning of the information. The equivalent word happens typically the reason behind incorrect within the vocabulary employed by the authors of documents and therefore the users of data retrieval systems. As a result, mathematician or keyword queries typically come back immaterial results and miss data that's relevant. LSI is additionally wont to perform automatic document categorization. The document categorization is that the assignment of documents to at least one or additional predefined classes supported their similarity to the abstract content of the classes. LSI uses example documents to ascertain the abstract basis for every class. Throughout categorization process, the ideas contained documents square measure classified to check with alternative ideas and a class assigned to the documents supported the similarities between the ideas.

\section{E-branding}

E-branding refers to the full of a company's values, proficiency, attitudes, vision, mission, temperament and look that's flaunted to the audience on-line. The final structure of E-Stigmatisation is shown in Figure three.10. Stigmatisation has been characterised because the method of making price through the supply of a desirable and protracted supply and client expertise that may satisfy customers and keep them coming. In our proposed system, the E-branding used as a marketing strategy for selling the product of the poly house. So the relevant information will be provided as the advertisement of the product in a social media. The branding technique provides the product details, quality, growth information, benefits of the product and the other related information will be provided. As customers develop trust in the brand through satisfaction of use and experience, and that have the opportunity to start building relationships with them, strengthening the brand further the how much of user using the product information to be provided.

\section{Conclusion}

In E-Branding, the whole leaders sometimes have the monetary strength to forestall competitors, and potential competitor's area unit sometimes disinclined to enter the market if existing brands satisfy customers. Therefore, modify a product to ascertain a novel identity and to extend the chance of attracting an oversized quantity of continual product. product with a history of robust brands area unit seemingly to keep up larger management over the balance of power between them and customers, and command a better market share and premium worth against generic, unbranded, equivalents. Strong, prosperous brands, thus, shift the competitive framework within the product's favour, giving it intangible, troublesome to duplicate, values with that to reinforce it's a lot of basic product, worth and distribution advantages. Although disapproval has attracted considerable analysis attention, its role and contribution to business performance have remained contentious. Whereas several analysts have foretold the departure of e-brands, others have argued that success on the web is all regarding disapproval. the previous dispute that brands have very little to contribute within the net marketplace and will, indeed, not be continuous because of the extent of worth clarity related to the web 
medium, the increasing convenience, through a number of easy keystrokes, of subtle search engines and product-comparison tools therefore products/services and willing sellers may be accessed on-line. It expands the client relationship. E-Branding offers straightforward survive of farmer among the competitors. It provides familiarity, loyalty and name to customers.

\section{References}

[1] P. Barnaghi and C. H Foh, Centre for Communication Systems Research Electronic Engineering Department University of Surrey Autumn Semester 2013/2014.

[2] K. E. Borade, C. S. Patil and R. R. Karhe, "Polyhouse Automation System", International Journal of Advanced Research in Computer Science and Software Engineering, vol. 3, no. 8, (2013) August, pp. 602-607.

[3] P. Jonnala and S. Satrasupalli, "Semi-Automated Polyhouse Cultivation using LabVIEW", International Journal of Computer Science and Business Informatics, ISSN: 1694-2108, vol. 7, (2013) November.

[4] K. Sachdeva, N. Kuma and R. Kumar, "Monitoring and Controlling of Environmental Parameters of Polyhouse Based On Lab VIEW", vol. 4, no. 9, (2015) September.

[5] www.googlelightweb.com.

[6] K. Gopala Krishna Pillai, "Glasshouse, Greenhouse and Polyhouse Crops", Mealybugs and their Management in Agricultural and Horticultural crops, (2016).

[7] S. S. Kulkarni and S. K. Gupta, "Android Application Development for Polyhouse Control”, Global Journal of Engineering Science and Research Management, (2016).

[8] R. Verma, S. J. Singh, R. K. Singh and S. Prakash, "Indexing of Viruses of Polyhouse Grown Capsicum (Capsicumannuuml.)", In Pune Causing Severe Symptoms and Epiphyrosis, (2004).

[9] K. Gopala Krishna Pillai, "Glasshouse, Greenhouse and Polyhouse Crops", Mealybugs and their Management in Agricultural and Horticultural crops, (2016).

[10] S. S. Kulkarni and S. K. Gupta, "Android Application Development for Polyhouse Control", Global Journal of Engineering Science and Research Management, (2016). 
International Journal of $u$ - and e- Service, Science and Technology Vol.10, No.10 (2017) 